\title{
COSTOS DE ATENCIÓN EN UCI DE UN HOSPITAL UNIVERSITARIO DE BOGOTÁ
}

\section{D.C.}

César Orlando Enciso Olivera MD*, Kennedy Arturo Guerra Urrego MD*, Mario Gómez Duque MD,FCCM**, Efraín Meneses Góngora MD,MAS***

\section{Resumen}

Se realizó un estudio descriptivo retrospectivo que evaluó el costo generado por la atención de los pacientes en una UCI de tipo mixto en un hospital universitario. El objetivo fue establecer de manera detallada cada uno de los recursos consumidos tomando la perspectiva del prestador de servicios.

Se evaluó la historia clínica de todos los pacientes atendidos durante el período comprendido entre el 1 de octubre de 2003 y el 31 de octubre de 2004, asignándolos de acuerdo con el diagnóstico primario que justificó el ingreso a la unidad en cinco grupos que denominamos de diagnóstico, relacionados de la siguiente manera: 1) cardiovasculares médicos, 2) cardiovasculares quirúrgicos, 3) sepsis de manejo médico, 4) sepsis de manejo quirúrgico y 5) el trauma. Se determinó el consumo de recursos a partir de la factura de venta, pero el valor aplicado corresponde al precio de compra del insumo.

Se incluyeron 190 pacientes de los cuales no fue posible completar la información financiera en 43 , por lo que al final el tamaño de la muestra fue de 147. En nuestro estudio el mayor consumo de recursos se encontró en aquellos que ingresaron por sepsis y en todos los grupos los que tuvieron mayor peso fueron el pago del recurso humano y en segunda instancia la nutrición, comportamiento que sigue la tendencia descrita en artículos escritos en países con alto desarrollo tecnológico, a pesar de las diferencias claras en los sistemas de atención en salud.

Conclusión: la distribución de los componentes de costos de atención en la UCI analizada concuerda con otros estudios extranjeros. El desarrollo en los sistemas de información y el conocimiento del costo detallado constituyen una herramienta indispensable para la toma de decisiones.

Palabras clave: costo, cuidado intensivo, recursos, tratamiento, distribución, contención de costos, inversión en salud.

Abreviaturas: UCI, unidad de cuidados intensivos; GDR, grupo de diagnóstico relacionado; APACHE II, Acute physiological and cronic health evaluation; DE, desviación estándar. EAPB, Empresas Administradoras de Planes de Beneficio.

\section{Introducción}

El consumo de elementos muy especializados aunado al hecho de que se requieran de manera permanente, así no se vayan a emplear en el momento, hacen que las UCI sean centros catalogados como de alto costo. Por esta razón, en cada una de las partes del sistema de salud y desde una perspectiva propia, se ha despertado un gran interés en investigar cómo

\section{Fecha recibido: abril 10 de 2006}

Fecha aceptado: mayo 15 de 2006

Residentes de medicina crítica, Fundación Universitaria de Ciencias de la Salud.

* Jefe de la unidad de cuidados intensivos, Hospital de San José. Jefe del programa de medicina crítica FUCS.

*** Jefe de la oficina de calidad y auditoría médica, Hospital de San José controlar el gasto generado por la atención de los pacientes críticos.

El ingreso a la UCI corresponde al $10 \%$ del total de hospitalizaciones en norteamérica. Este porcentaje de la estancia muestra gran desproporción cuando al evaluar el costo se encuentra que representa alrededor de $30 \%$ del total del monto generado por la atención de todas las causas de hospitalización ${ }^{1,2}$ y que a manera de ejemplo, en 1984 alcanzó una magnitud equivalente al $1 \%$ del producto interno bruto en norteamérica. ${ }^{1,2}$

Este fenómeno ha llevado a considerar si es justificable la inversión de estas fuertes sumas de dinero en la atención de unos pocos pacientes, cuyas pato- 
logías no siempre han podido ser atendidas con un resultado satisfactorio. ${ }^{3}$ Sin embargo, independiente del beneficio que el cuidado intensivo tenga en la calidad de vida de quienes egresan de las unidades, esta disciplina sigue una tendencia creciente durante los últimos años y forma parte de los adelantos que han favorecido el desarrollo de otras áreas como la cirugía ya sea con el cuidado postoperatorio de pacientes sometidos a procedimientos mayores que antes sucumbían en las siguientes horas de la intervención o por permitir que los enfermos críticos puedan ser estabilizados, para luego ser sometidos a procedimientos que constituyan la terapia definitiva. ${ }^{4}$

El crecimiento se ve representado cuando se analiza el comportamiento que ha tenido el cuidado intensivo en los Estados Unidos, donde en 1991 la Asociación Americana de Hospitales informó un número aproximado de $2.876 \mathrm{UCI}$ con un total de 32.850 camas y en 1997 el número de unidades no coronarias ya había llegado a 5.979 con 72.500 camas, lo que implica aumentos equivalentes a $51.9 \%$ y $54.7 \%$ respectivamente. Este fenómeno por obvias razones ha magnificado el costo pero no de la manera proporcional que se supondría, sino con un valor muy superior determinado por la aplicación de nuevas tecnologías cada vez más costosas. ${ }^{5,6}$

En otras palabras, a pesar de un beneficio no tan claro en todos los grupos de pacientes, las unidades de terapia intensiva tienen un espacio cada vez más amplio en la medicina actual y se hace necesaria de manera urgente la aplicación de estrategias de control de costos en las mismas. ${ }^{7}$

Como una primera aproximación que facilite la comprensión del tema, discriminamos los tipos de análisis de costos que pueden realizarse en los siguientes tres grupos: ${ }^{8}$

1. Análisis de costo efectividad: busca comparar una opción que se emplea de manera frecuente (de base) con una alternativa y determinar la magnitud del cambio producido sobre los costos.
2. Análisis de identificación o variación de costos: describe el comportamiento del costo en un período sin relacionarlo con el desenlace ni realizar comparación entre terapias.

3. El tercer tipo de trabajo corresponde a los estudios de costo beneficio: el análisis de un costo generado por una intervención con respecto al beneficio obtenido en un desenlace determinado. En general, son estudios con un mayor grado de dificultad, puesto que la medición del beneficio se basa en escalas más complejas como son los años de vida potencialmente perdidos, los de vida saludable, la calidad de vida, etc.

Otro aspecto para tener en cuenta en los análisis de costos es la perspectiva desde la cual se realizan. En este punto es obvia la diferencia entre cada una de las partes que participan en la atención de un paciente, quienes de acuerdo con la visión del problema pueden tener un interés diferente e incluso opuesto.

Se pudiera considerar de acuerdo con esta situación que en un sistema de salud aparece una interrelación entre cuatro actores: ${ }^{8,9}$ el paciente que constituye el centro de atención; un proveedor del servicio, que en la ejecución de su labor genera un costo variable, cuantificable y susceptible de ser relacionado con los resultados obtenidos como un indicador de eficiencia; un pagador, que de acuerdo con el tipo de vinculación del paciente puede ser un intermediario entre los estamentos gubernamentales y el proveedor, ya sea una empresa de carácter privado, con menor frecuencia el mismo paciente o el estado que asume el papel de regulador de las interrelaciones planteadas.

Es evidente, de acuerdo con esta estructura, que los objetivos, los indicadores y los intereses de cada una de las partes son diferentes. Su interacción se resume así: un paciente o grupo de pacientes que requieren un servicio cuyo interés principal es reestablecer su salud, un proveedor de servicios que tiene por finalidad optimizar sus recursos para lograr cada día una mejor atención a un menor costo, lo 
cual dentro de la estructura lo posicionaría ventajosamente en el mercado, y un pagador que tiene un interés primordial en cubrir con la mínima cantidad de recursos la mayor población posible. Como lo expusimos antes, existe la probabilidad de que este pagador corresponda al mismo paciente, caso en el cual sus intereses serán dobles tratando de obtener el mayor beneficio con el menor costo posible.

La perspectiva que se tome como directriz del trabajo es muy importante dado que el valor que se utiliza como costo varía de acuerdo con ella. ${ }^{8}$ Así, para el proveedor de servicios el costo de producción es el valor más importante y sus estrategias buscan reducirlo para lograr un mayor margen de ganancia y para el pagador el costo de compra del servicio se constituiría en el punto más significativo, pues de manera comparativa puede determinar cuales proveedores le muestran mejores indicadores de eficiencia.

En lo referente al costo de atención por enfermo las conclusiones son muy variables, determinadas por las diferencias inherentes al tipo de pacientes, sus diagnósticos y las creencias, actitudes y prácticas de cada grupo de cuidado intensivo.

Noseworthy, en Canadálevaluó de manera prospectiva en una unidad de tipo mixto el costo de atención de 690 pacientes y encontró un promedio de C\$ $1.508(+-475)$ por cada uno de ellos. Dentro de este grupo se realizó una técnica de microcosteo llegando a discriminar los minutos de atención por cada uno de los miembros del grupo de cuidado intensivo, excluyendo de su población los postoperatorios de cirugía mayor no complicada que solo requerían monitoreo. El costo por estancia fue de C\$ 7.520 (+- 11.606) y no se encontró una relación entre el costo y la edad o la severidad expresada por medio del APACHE II.

El impacto de un entorno distinto durante el estudio de costos puede verse reflejado en la magnitud de la diferencia en los resultados de un trabajo similar desarrollado por Chirag $\mathrm{P},{ }^{10}$ quien con una metodología comparable evaluó el costo de una UCI en la
India. Encontró que el valor promedio por paciente era de 57 dólares por día, que corresponde apenas al $3.78 \%$ del valor en el estudio de Noseworthy. Las conclusiones pueden ofrecer alguna dificultad por las diferencias en los grupos de pacientes, si se tiene en cuenta que el hospital de Bombay donde se realizó el estudio es un centro de atención neurológica y neuroquirúrgica, con una tendencia a mayor mortalidad con respecto a otras poblaciones de cuidado intensivo. Sin embargo, los autores relacionan esta mortalidad de $36 \%$ en su grupo de pacientes contra los reportados como mortalidad en los Estados Unidos, que no sobrepasa el 25\%, con la limitación de recursos tecnológicos y humanos y se cita como ejemplo el número de pacientes atendidos por enfermera que correspondía al doble del asignado en Estados Unidos.

A pesar de que muchos sesgos pueden acompañar estas comparaciones, la diferencia de inversión per cápita entre Estados Unidos y países en vía de desarrollo como el nuestro, es muy significativo. Este fenómeno ocasiona una gran dificultad en la aplicación de las conclusiones de la mayor parte de trabajos de costos en nuestro entorno. Además, consideramos que es importante evaluar las principales causas de error en los trabajos de costos, luego de encontrar en la literatura el análisis que con respecto al tema desarrolló en 1995 Gyldmark et al. ${ }^{8}$

En este trabajo se practicó una búsqueda de los artículos escritos en el idioma inglés que analizaran el costo en pacientes adultos críticos y que describieran en detalle la metodología de aproximación. Después de evaluarlos se describieron como las principales causas de error:

1. La no discriminación del costo de la UCI de manera independiente con el de después del egreso. Esto queda representado en el rango que se establece en el promedio de costo por paciente que osciló entre 1.783 y 46.835 dólares.

2. Falta de definición del valor que fue considerado como costo. Anteriormente se expuso este 
tópico cuando se describió la importancia de la perspectiva a partir de la cual se elabora el estudio.

3. El cálculo del costo por paciente basado en el total en un período, dividido por el número de enfermos atendidos. Este método se describió como una forma de aproximar el costo desde la perspectiva gubernamental en busca de realizar una asignación gruesa del recurso, pero en el caso de un área específica como el cuidado crítico se pierde el rigor metodológico, dado que la diversidad en patologías y la diferencia en el valor de la terapia no permite utilizar una aproximación tan general al costo.

4. Falta de la descripción de la metodología con la cual se recogió la información, relativa a la descripción puntual de la ubicación temporal del trabajo y el sistema empleado en la recolección, es decir si se tomó sobre las facturas de venta, los cargos en un sistema de información, etc.

5. Promediar el costo dividiendo su monto total por los días de estancia, sin tener en cuenta que de acuerdo con la gravedad de la enfermedad y la respuesta a la terapia, el costo puede variar a lo largo de los días sin seguir una tendencia uniforme durante la hospitalización.

El análisis de este último trabajo permite determinar que el costo debe ser cuantificado de una manera individual y no promediada, en especial cuando se evalúa el costo por día. Resalta la importancia de determinar una perspectiva clara desde la cual se desarrollará el trabajo y describe minuciosamente la metodología que se sigue en la recolección de la información. Todos estos datos se encuentran aplicados en la descripción metodológica. ${ }^{8,9}$

De acuerdo con esta descripción, el presente trabajo se ubica dentro de un análisis tanto de identificación como de variación del costo mediante una metodología descriptiva, retrospectiva y sin pretender comparar nuestros costos con un desenlace particular. No se obtuvo financiación de empresas farmacéuticas ni hay intención de favorecer una práctica específica.

\section{Materiales y métodos}

La UCI en la cual se desarrolló el estudio corresponde a una unidad de tipo mixto, en donde son atendidos pacientes adultos con patología médica o quirúrgica, dentro de un sistema semiabierto en el cual las decisiones son tomadas por el especialista en medicina crítica, pero con la participación del médico de la especialidad que hospitaliza el paciente. Consta de diez camas dentro de un hospital de tipo universitario con 240 camas.

Se revisaron todas las historias clínicas correspondientes a la estancia en la UCI de los pacientes atendidos durante el perÍlodo comprendido entre el 1 de octubre de 2003 y el 31 de octubre de 2004. La información obtenida fue consignada en una base de datos Microsoft ACCESS XP ${ }^{\circledR}$ en la cual se almacenó el conjunto de datos correspondiente a los costos y después por medio de consultas cruzadas, se enlazó con la información clínica.

Una vez evaluada la historia clínica, los pacientes fueron asignados de acuerdo con el diagnóstico principal de ingreso, en uno de cinco grupos que para el efecto se denominaron GDR, con el fin de obtener unas categorías homogéneas con respecto al tipo de recursos consumidos, sin tener en cuenta en esta clasificación el puntaje de severidad que fue considerado entre las variables demográficas y calificado por medio del APACHE II. ${ }^{11}$

La clasificación por GDR que se empleó fue la siguiente:

1. Pacientes cardiovasculares en tratamiento médico: se incluyeron aquellos con patología de este sistema orgánico que no requiriera intervención diferente a la farmacológica. Se encuentran agrupados aquí los que presentaron enfermedad coronaria, descompensación de falla cardíaca, arritmias y crisis hipertensivas que requirieron manejo por agudización de su afección en la UCI.

2. Pacientes cardiovasculares en tratamiento quirúrgico: los que habían sido sometidos a un pro- 
cedimiento electivo o de emergencia por parte de cirugía cardiovascular o vascular periférica. También se incluyeron los que hubieran sido sometidos a procedimientos por hemodinamia como angioplastia con o sin stent y la implantación de marcapasos definitivos.

3. Pacientes con sepsis de tratamiento médico: los que tuvieran diagnóstico de infección como causa de ingreso a la unidad y que como parte de su tratamiento no requirieron desbridamiento o drenaje.

4. Pacientes con sepsis de manejo quirúrgico: corresponde a los que ingresan con un diagnóstico primario de infección, pero que requirieron como parte del tratamiento ser intervenidos quirúrgicamente para drenaje o desbridamiento, además del tratamiento antimicrobiano.

5. Pacientes con diagnóstico de trauma: se asignaron a este grupo los que ingresaron a la unidad con una patología traumática como diagnóstico principal. Se incluyeron aquellos con trauma craneoencefálico dado que no se obtuvo un número suficiente que permitiera el análisis específico en este tipo de pacientes.

Se excluyeron de la selección a los siguientes enfermos:

- Estancia inferior a 48 horas en la unidad.

- Con patología oncológica incluyendo quienes ingresaron por neutropenia febril.

- En postoperatorio de cirugía electiva de una especialidad diferente a la cardiovascular, que hubieran ingresado para extubación programada o monitoreo postoperatorio.

- Quemados no infectados.

- Quienes fueron remitidos a otro centro.

- Con complicación de una enfermedad inmunológica.
- Menores de 16 años.

Se excluyeron aquellos pacientes con estancia inferior a 48 horas, con el fin de reflejar el costo de acuerdo con el promedio de estancia en la unidad que corresponde a 3,4 días. Así mismo, los pacientes que se remitieron a otro centro no se tuvieron en cuenta, dado que no se contó con información relativa a la evolución y desenlace de su enfermedad.

Los demás criterios de exclusión corresponden a afecciones de baja frecuencia en la unidad o a enfermedades que ameritan un análisis específico del costo como son las hematológicas, dentro de las cuales la causa más frecuente de ingreso a la unidad es la neutropenia febril, con las implicaciones económicas que son conocidas en este grupo de pacientes. Los menores de 16 años fueron atendidos en la unidad pediátrica de la institución.

Para la recolección de la información correspondiente al costo, se optó por la perspectiva del prestador del servicio de lo cual se deriva que el interés primordial es conocer el generado por el consumo de los recursos. Por esta razón, la información detallada se tomó de los ítems consignados en la factura emitida para la EAPB y el valor aplicado corresponde al valor del costo de adquisición del insumo, el cual nos fue suministrado por el departamento de compras de la institución, en pesos colombianos.

Con respecto al costo por el talento humano, al principio se planteó la opción de utilizar el puntaje de intervención representado por el Therapeutic Index Score Survey (TISS) ${ }^{28}$, pero los datos de acuerdo con los minutos consumidos según esta aplicación, representados en pesos, no corresponden al valor real que se empleó en el recurso humano. Por este motivo se ajustó el valor aportado por el personal tomando como parámetros el porcentaje de ocupación y el número de pacientes atendidos al mes, sin olvidar que la UCI cuenta con una planta fija de auxiliares de enfermería, enfermeras licenciadas, médicos, nutricionista y terapeuta respiratoria que, independiente del número de pacientes hospitalizados, se encuentra asignado de manera permanente y genera un costo 
fijo cuyo valor por paciente puede variar de acuerdo con el número atendido. La fórmula utilizada fue la división del costo total por el porcentaje de ocupación teniendo en cuenta que este rubro está asignado a una ocupación del $100 \%$ y luego dividir el valor obtenido entre el número de personas atendidas durante ese mes. Esta última cifra se consideró como el valor corregido por paciente según la ocupación y con este dato se calculó el valor por paciente durante el mes evaluado. Para la asignación por día, el valor correspondiente al paciente se dividió por el número de días de su atención e incluyó el rubro de personal. Las ecuaciones empleadas fueron las siguientes:

Todos los valores se expresaron en pesos corrientes de 2004 y su costo fue corregido si se modificaba su precio durante el mes en que se realizó la compra.

Costo total de la nómina

Costo corregido de la UCI por mes por ocupación Porcentaje de ocupación de la UCI en fracción decimal durante el mes

Costo corregido por

Costo corregido por paciente la ocupación Número de pacientes atendidos durante el mes

Costo corregido por

Costo por día por paciente paciente

Número de días de estancia

Los datos obtenidos fueron llevados a las tablas por medio de la aplicación diseñada en Microsoft ACCESS XP ${ }^{\circledR}$, para tal fin.

\section{Análisis de los datos}

Las variables primarias como la edad, el puntaje de APACHE II en las primeras 24 horas, la estancia en la unidad y el desenlace se representan como la mediana y la DE. En el análisis del costo se presenta la media aritmética y los límites mínimo y máximo de la serie expresando en porcentaje la participación de cada uno de los grupos con respecto a la totalidad de la muestra.

\section{Resultados}

Se seleccionó un total de 190 pacientes de acuerdo con los criterios establecidos. De estos no fue posible obtener la información completa de 43 que habían sido asignados de la siguiente manera: al grupo 1, cuatro pacientes, al grupo 2 , diez pacientes, al grupo 3 , trece pacientes y ocho a grupos 4 y 5 . Estos pacientes se excluyeron de la muestra quedando la población de estudio conformada por 147 individuos cuya distribución se presenta en la Tabla 1.

El mayor número de pacientes se encuentra en los grupos quirúrgicos, lo cual representa la estadística general de la unidad. La distribución de la edad no difiere de las características epidemiológicas descritas en la literatura, con predominio de la enfermedad coronaria en el sexo masculino y mayores de 55 años,y con el grupo más joven en los que ingresaron por patología traumática.

El promedio de estancia por grupos no mostró un distanciamiento notorio con respecto del promedio general de estancia en la UCI (3,4 días). En el grupo I se presentó un aumento del intervalo de la DE derivado de la prolongada estancia de uno de sus pacientes, quien ingresó por una patología cardiovascular médica correspondiente a un bloqueo auriculoventricular, con un evento isquémico miocárdico complicado y disfunción ventricular durante su estancia en la unidad (Tabla 2).

En relación con las condiciones clínicas de la población de interés, como ya se mencionó, se utilizó el puntaje APACHE II $^{11}$ determinado dentro de las primeras 24 horas de estancia de cada paciente. En la unidad esta escala se realiza en todos los pacientes de manera diaria con fines de establecer parámetros de comparación, por lo cual incluso los de los grupos de enfermedad coronaria tienen asignado un puntaje, sin que en esta patología haya sido validada como he- 
Tabla I. Características demográficas de la población

\begin{tabular}{l|c|c|c|c|c|c} 
& Mujeres & Hombres & \% Participación por grupo & Mediana de la edad & Límite inferior & Límite superior \\
\hline Grupo 1 & 8 & 19 & 18,37 & 62 & 39 & 91 \\
\hline Grupo 2 & 12 & 24 & 24,49 & 56.5 & 33 & 87 \\
\hline Grupo 3 & 8 & 9 & 11,56 & 54 & 16 & 92 \\
\hline Grupo 4 & 25 & 14 & 26,53 & 60 & 23 & 85 \\
\hline Grupo 5 & 7 & 21 & 19,05 & 42 & 21 & 78 \\
\hline Total & 60 & 87 & 100 & 55 & &
\end{tabular}

Fuente: análisis de datos.

Tabla 2. Características de la estancia(en días)

\begin{tabular}{|c|c|c|c|}
\hline & $\begin{array}{c}\text { Mediana de } \\
\text { estancia }\end{array}$ & $\begin{array}{c}\text { Límite } \\
\text { inferior }\end{array}$ & $\begin{array}{c}\text { Límite } \\
\text { superior }\end{array}$ \\
\hline Grupo 1 & 4 & 3 & 73 \\
\hline Grupo 2 & 4 & 3 & 47 \\
\hline Grupo 3 & 4 & 3 & 8 \\
\hline Grupo 4 & 4 & 3 & 23 \\
\hline Grupo 5 & 4 & 3 & 23 \\
\hline
\end{tabular}

Fuente: análisis de datos.

rramienta de predicción de mortalidad. El resultado discriminado por grupos se presenta a continuación (Tabla 3).

En la Tabla 4 se presenta la distribución de la mortalidad en cada uno de los grupos, resaltando el predominio en los pacientes víctimas de trauma, que como se observó en las características demográficas corresponde a la población más joven. Es de anotar que los datos de mortalidad no fueron objeto de correlación y constituyen información adicional para ampliar el contexto del estudio (Tabla 4).

\section{Tabla 4. Porcentaje de mortalidad}

\begin{tabular}{l|c} 
& Mortalidad (\%) \\
\hline Grupo I & 7,4 \\
\hline Grupo 2 & 22,2 \\
\hline Grupo 3 & 23,52 \\
\hline Grupo 4 & 25,64 \\
\hline Grupo 5 & 35,7
\end{tabular}

Fuente: análisis de datos.
Tabla 3. Resultados del APACHE II por grupos

\begin{tabular}{|c|c|c|c|c|}
\hline & $\begin{array}{l}\text { Puntaje } \\
\text { promedio }\end{array}$ & Mediana & $\begin{array}{l}\text { Puntaje } \\
\text { mínimo }\end{array}$ & $\begin{array}{l}\text { Puntaje } \\
\text { máximo }\end{array}$ \\
\hline Grupo 1 & 14 & 12,5 & 0 & 30 \\
\hline Grupo 2 & 26,5 & 31 & 0 & 51 \\
\hline Grupo 3 & 23,1 & 22 & 0 & 52 \\
\hline Grupo 4 & 26,1 & 27 & 6 & 53 \\
\hline Grupo 5 & 18,7 & 15 & 6 & 44
\end{tabular}

Fuente: análisis de datos.

La evaluación del índice TISS 28, cuya aplicación original es la asignación del recurso humano de acuerdo con el número de intervenciones y la complejidad de las mismas, mostró un valor más alto en los pacientes quirúrgicos, lo cual se encuentra relacionado con un mayor número de actividades. Los valores obtenidos no fueron empleados como herramienta en el cálculo del valor del recurso humano como se planeó inicialmente, por la diferencia significativa cuando se comparan los datos obtenidos al convertir en minutos y luego en pesos los puntos de TISS 28, con el valor real que se había generado por concepto del pago de personal. Los valores se exponen como información que permite evaluar la gravedad de los pacientes (Figura 1).

El gran total arrojado por costo global de tratamientos administrados, incluyendo complementación diagnóstica y terapéutica a la población de interés en el período de estudio, ascendió a \$455.496.193,00 que determina un valor promedio de atención por paciente de $\$ 3.098 .613,00$. En la Tabla 5 además se puede observar la distribución del costo por paciente en cada uno de los grupos, así como las cifras que 


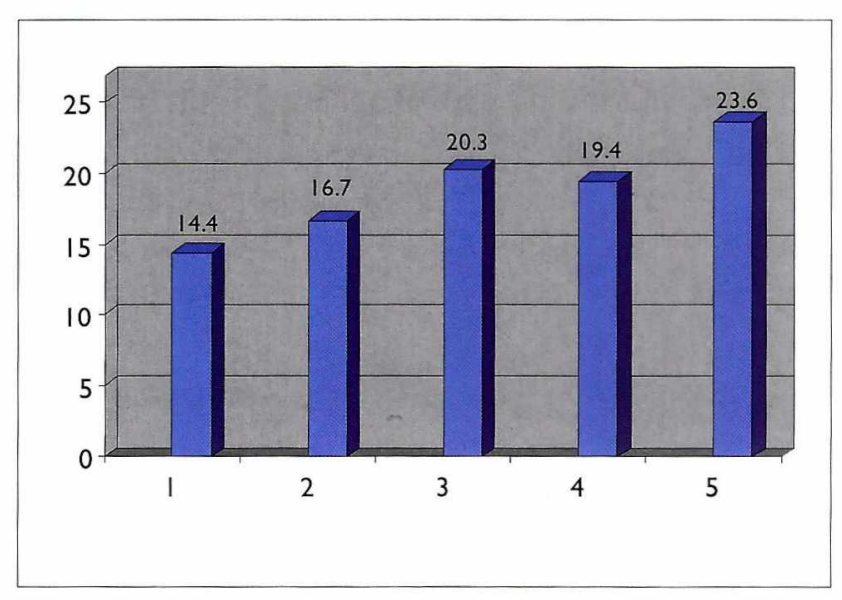

Figura I. Promedio de puntaje TISS 28 por grupo. limitan este valor, denotando una leve ventaja en los que fueron atendidos por patologías de índole infecciosa.

Respecto a la participación de cada componente de la atención, se obtuvo una distribución que evidencia una mayor concentración del costo sobre dos ítems: el de recursos humanos y la complementación terapéutica, representada por los costos derivados de la nutrición. Es de notar que el presente estudio concuerda con resultados de trabajos extranjeros en cuanto a la magnitud de la participación del costo del personal asistencial de la UCI. La (Tabla 6).

\begin{tabular}{|c|c|c|c|c|c|c|}
\hline & $\begin{array}{c}\text { Costo } \\
\text { global grupo }\end{array}$ & $\begin{array}{l}\text { Participación } \\
\text { \% por grupo }\end{array}$ & $\begin{array}{c}\text { Costo promedio } \\
\text { paciente }\end{array}$ & $\begin{array}{c}\text { Costo paciente } \\
\text { / día }\end{array}$ & Mínimo & Máximo \\
\hline Grupo I & $\$ 59.120 .504,00$ & 12,98 & $\$ 2.189 .648,30$ & $\$ 316.423,16$ & $\$ 846.976,00$ & $\$ 6.524 .539,00$ \\
\hline Grupo 2 & $\$ 92.712 .505,00$ & 20,35 & $\$ 2.575 .347,36$ & $\$ 444.025,41$ & $\$ 887.732,00$ & $\$ 9.135 .658,00$ \\
\hline Grupo 3 & $\$ 63.667 .530,00$ & 13,98 & $\$ 3.745 .148,82$ & $\$ 796.840,18$ & $\$ 1.505 .097,00$ & $\$ 9.230 .200,00$ \\
\hline Grupo 4 & $\$ 150.266 .445,00$ & 32,99 & $\$ 3.852 .985,77$ & $\$ 642.164,29$ & $\$ 1.312 .844,00$ & $\$ 13.713 .350,00$ \\
\hline Grupo 5 & $\$ 89.729 .209,00$ & 19,7 & $\$ 3.204 .614,61$ & $\$ 628.355,81$ & $\$ 1.123 .931,00$ & $\$ 7.997 .679,00$ \\
\hline Total & $\$ 455.496 .193,00$ & & & & & \\
\hline
\end{tabular}

Fuente: análisis de datos.

\begin{tabular}{|l|c|c|c|c|c|}
\hline \multicolumn{7}{|c}{ Tabla 6. Distribución de los componentes del costo por grupo (\%) } \\
\hline & Grupo I & Grupo 2 & Grupo 3 & Grupo 4 & Grupo 5 \\
\hline Analgesia & 0,13 & 0,42 & 0,11 & 0,28 & 0,3 \\
\hline Antibióticos & 6,98 & 2,02 & 14,57 & 14,49 & 3,69 \\
\hline Anticoagulación & 5,7 & 1,93 & 5,98 & 0,56 & 0,4 I \\
\hline Cardiovascular & 6,38 & 19,95 & 4,62 & 6,1 I & 7,43 \\
\hline Costo nutrición & 13 & 7,47 & 23,1 & 30,55 & 24,27 \\
\hline Costo personal UCI & 42,9 & 37,12 & 26,36 & 24,62 & 30,59 \\
\hline Digestivo & 2,68 & 0,53 & 2,58 & 0,81 & 0,5 \\
\hline Endocrino & 0,08 & 0,01 & 0,07 & 0,05 & 0,28 \\
\hline Insumos & 5,02 & 4,73 & 3,3 & 5,12 & 4,3 \\
\hline Monitoreo & 7,37 & 7,83 & 5,13 & 5,27 & 5,39 \\
\hline Sedación & 4,69 & 4,46 & 3,56 & 3,73 & 9,74 \\
\hline Soluciones & 2,55 & 3,74 & 3,21 & 4,27 & 4,71 \\
\hline Soporte renal & 0,19 & 0,16 & 0,13 & 0,12 & 0,07 \\
\hline TOTAL & 100 & 100 & 100 & 100 & 100 \\
\hline
\end{tabular}

Fuente: análisis de datos. 
muestra la distribución detallada de la participación de cada ítem dentro de la composición global de costos por grupo.

El costo generado por los recursos diagnósticos asciende a $\$ 53.493 .850,00$, que corresponde al $11,7 \%$ del costo de las intervenciones terapéuticas. Cuando se analiza el comportamiento dentro de los grupos se encuentra que la relación entre la inversión en recursos diagnósticos con respecto al total del costo, tuvo el comportamiento observado en la

Tabla 7.

Tabla 7. Participación de recursos diagnósticos

\begin{tabular}{l|l}
\hline Grupo I & $7 \%$ \\
\hline Grupo 2 & $5,2 \%$ \\
\hline Grupo 3 & $16 \%$ \\
\hline Grupo 4 & $11,5 \%$ \\
\hline Grupo 5 & $11 \%$
\end{tabular}

Fuente: análisis de datos.

\section{Discusión}

La magnitud del costo generado por la atención del paciente crítico se ve reflejada en el presente trabajo de manera muy similar a las investigaciones que se han realizado en otros países, ${ }^{1}$ tanto en el análisis de los atendidos en unidades de tipo mixto con diversas enfermedades como en trabajos enfocados a patologías específicas como la sepsis, ${ }^{12}$ teniendo en cuenta que los datos demográficos no difieren en forma significativa de los reportados por estos autores.

Acerca de los datos proporcionados en el análisis demográfico, la distribución por grupos etáreos es congruente con la epidemiología de las enfermedades que se analizaron, con predominio de la enfermedad coronaria en pacientes de sexo masculino por encima de los 55 años y del trauma en los más jóvenes, resaltando una mortalidad por encima del promedio de la unidad en este último grupo, que amerita la realización de trabajos posteriores con un enfoque específico en la patología traumática, que no es el objeto del presente estudio. En el análisis de los puntajes de severidad cabe anotar que el puntaje APACHE II informado corresponde al realizado a las 24 horas del ingreso a la unidad. Esta escala es realizada a diario en la UCI más con el fin de establecer parámetros de comparación que como predictor de mortalidad, por esta razón incluso los pacientes coronarios en quienes no se validó esta herramienta en el trabajo original del doctor Knaus tienen asignado el puntaje.

En el análisis de la distribución del costo por grupo de diagnóstico, las enfermedades infecciosas que corresponden a los grupos 3 y 4 constituyen la patología que genera el mayor consumo de recursos, tendencia que ha sido descrita en otros trabajos. Vale la pena aclarar que el tratamiento de la sepsis en la unidad ha sido orientado por guías que buscan obtener una reanimación temprana, con indicadores de la perfusión global por medio de la saturación venosa de oxígeno como meta hemodinámica de la reanimación y el drenaje del foco infeccioso como premisa fundamental del tratamiento. En el análisis específico de la distribución de los recursos en este grupo, llama la atención el bajo rubro invertido en el grupo de antimicrobianos. Se cree puede estar relacionado con las políticas de control de resistencia microbiana con la aplicación de terapia de reducción programada del espectro antibiótico y de control de la infección nosocomial. El análisis específico de esta correlación no fue objeto de este estudio.

Con respecto a la distribución del costo entre los diferentes tópicos que comprenden la atención, fue un hallazgo sorpresivo que pese a las diferencias entre los sistemas de salud de los países, la inversión en el recurso humano corresponde al principal rubro en cuatro de los grupos de diagnóstico, estableciendo que la inversión en el talento humano constituye un factor muy importante de desarrollo en la prestación de servicios.

El segundo tópico en importancia fue el soporte nutricional, el cual tiene incluso un valor superior al recurso humano en el grupo de pacientes con sepsis de manejo quirúrgico. Esta situación se ha considerado relacionada con las guías de nutrición temprana 
que se siguen en la unidad con un predominio del soporte por vía enteral siempre que sea posible. La diferencia en el grupo de sepsis quirúrgica, en el cual el rubro de nutrición es superior a todos los demás elementos, la hemos relacionado con un inicio tardío del soporte nutricional enteral por su condición clínica o postoperatoria, recibiendo soporte parenteral durante los primeros días de estancia en la unidad. El mayor costo de este último ha sido establecido en la mayoría de los trabajos al comparar las dos rutas para soporte nutricional, constituyendo la principal evidencia a favor de la vía enteral, sin que aun se tengan datos concluyentes con respecto al beneficio en morbilidad y mortalidad a favor de alguna de las técnicas.

El costo de la nutrición es además un factor de relevancia cuando se tiene en cuenta que la mayoría de productos nutracéuticos no se encuentran incluidos en el plan obligatorio de salud y que en algunas ocasiones son objeto de glosa por parte de las EAPB. Este factor debe ser analizado dentro de las políticas contractuales por el impacto financiero que puede tener desde la perspectiva del prestador de servicios.

Por último, la evaluación de la participación de los estudios diagnósticos dentro del total del costo, no superó en ninguno de los grupos el $16 \%$, relacionando este dato con el hecho de que se ha abolido el protocolo de solicitud de exámenes de rutina en la unidad y quedando a criterio del médico tratante ordenar las pruebas que considere pertinentes para el seguimiento de las condiciones clínicas del paciente, con la consideración que la solicitud de pruebas en busca de anormalidades sin soporte clínico solo ofrecen un sobrecosto en la atención del paciente crítico sin ninguna ventaja en la evolución.

\section{Conclusiones}

A pesar de las diferencias en los sistemas de atención de salud y de las políticas contractuales entre los países desarrollados y en vía de desarrollo como el nuestro, las patologías de origen infeccioso constituyen las enfermedades que consumen la ma- yor cantidad de recursos, La inversión en el talento humano es el rubro más importante en nuestro trabajo seguido del soporte metabólico y nutricional.

Es imperativo el desarrollo de sistemas propios y cada vez más detallados de información que permitan una toma de decisiones orientada por parámetros objetivos, con mayor probabilidad de éxito de las políticas contractuales y de asignación de recursos.

\section{Referencias}

1. Noseworthy TW, Konopad E, Shustack A, Johnston R, Grace M. Cost accounting of adult intensive care: methods and human and capital inputs. Crit Care Med. 1996 Jul;24(7):1168-72.

2. Brilli RJ, Spevetz A, Branson RD, Campbell GM, Cohen H, Dasta JF, Harvey MA, Kelley MA, Kelly KM, Rudis MI, St Andre AC, Stone JR, Teres D, Weled BJ; American College of Critical Care Medicine Task Force on Models of Critical Care Delivery. The American College of Critical Care Medicine Guidelines for the Defintion of an Intensivist and the Practice of Critical Care Medicine. Critical care delivery in the intensive care unit: defining clinical roles and the best practice model. Crit Care Med. 2001 Oct;29(10):2007-19.

3. Glance LG, Osler T, Shinozaki T. Intensive care unit prognostic scoring systems to predict death: a cost-effectiveness analysis. Crit Care Med. 1998 Nov;26(11):1842-9.

4. Atkinson S, Bihari D, Smithies M, Daly K, Mason R, McColl I. Identification of futility in intensive care. Lancet. 1994 Oct 29;344(8931):1203-6.

5. Ridley S. Biggam M, Shone P: A cost-benefit analysus of intensive therapy. Anaesthesia 1993; 48:14-19.

6. Joint Position Statement: Essential provisions for critical care in health system refor. Crit Care Med 1994; 22:20172019.

7. Esserman L, Belkora J, Lenert L. Potentially ineffective care. A new outcome to assess the limits of critical care. JAMA. 1995 Nov 15;274(19):1544-51.

8. Gyldmark M. A review of cost studies of intensive care units: problems with the cost concept. Crit Care Med. 1995 May;23(5):964-72.

9. Heyland DK, Gafni A, Kernerman P, Keenan S, Chalfin D. How to use the results of an economic evaluation. Crit Care Med. 1999 Jun;27(6):1195-202.

10. Parikh CR, Karnad DR. Quality, cost, and outcome of intensive care in a public hospital in Bombay India: Crit Care Med. 1999 Sep;27(9):2032-3.

11. Knaus WA, Draper EA; Wagner DP, et al: APACHE II: Severity of disease classification system. Crit Care Med 1985; 13:818-829.

12. Burchardi H, Schneider H. Economic aspects of seversepsis: a review of intensive care unit costs, cost of illness and cost effectiveness of therapy. Pharmacoeconomics. 2004;22(12):793-813. 